\title{
Ultrasonographic Assessment of Costochondral Cartilage for Microtia Reconstruction
}

\author{
Jessa E. Miller, BS (D; Janice L. Farlow, MD, PhD; Elizabeth M. Knecht, MD; Ramon Sanchez, MD; \\ Jennifer C. Kim, MD; David A. Zopf, MD, MS
}

\section{INTRODUCTION}

Microtia is a congenital abnormality of the auricle, which ranges in severity from mild structural defects to complete absence of the ear (anotia). ${ }^{1}$ The prevalence of microtia is estimated to be 0.83 to 17.4 per 10,000 births. ${ }^{1}$ Several studies have shown that individuals with microtia have difficulty with social integration and lack self-confidence. ${ }^{2-4}$ Microtia poses a functional challenge for children who require eyeglasses, and the prevalence of mood disorders such as depression, interpersonal sensitivity, social difficulties, hostility, and aggression increases with age among patients with unrepaired microtia. ${ }^{5}$ Studies suggest that children become aware of their physical differences between the ages of 3 and 4 years; however, psychological trauma is most likely to occur in the first grade, when children begin to socialize with a large group of peers for the first time.,5

One of the mainstays of treatment for congenital microtia is reconstruction using autologous costochondral cartilage, first described by Tanzer ${ }^{6}$ and subsequently modified by Cronin, Brent and Nagata (Patel et al.). ${ }^{7}$ Despite decreased morbidity and mortality over time, the procedure is still associated with complications including chest wall deformity, pneumothorax, hematoma, infection, overlying soft tissue necrosis, cartilage framework exposure, and graft resorption or loss. ${ }^{7}$ The risk of chest wall deformity and insufficient cartilage for auricular reconstruction in particular have been shown to increase dramatically if costochondral cartilage harvesting is done too early in a child's development., ${ }^{8,9}$ Thompson et al. reported chest wall deformity rates of

From the University of Michigan Medical School (J.E.M.), Ann Arbor, Michigan; Department of Otolaryngology-Head and Neck Surgery (J.L.F., E.M.K., J.C.K., D.A.Z.); Department of Radiology (R.s.), Michigan Medicine, Ann Arbor, Michigan, U.S.A.

Editor's Note: This Manuscript was accepted for publication on May $29,2018$.

The authors have no funding, financial relationships, or conflicts of interest to disclose.

Send correspondence to David A. Zopf, MD, Department of Otolaryngology-Head and Neck Surgery, Michigan Medicine, C. S. Mott Children's Hospital, 1540 East Hospital Drive, SPC 4241, Ann Arbor, MI 48109. E-mail: davidzop@med.umich.edu

DOI: 10.1002/lary.27390
$33 \%$ and $8 \%$ for patients who underwent cartilage harvest between the ages of 2 to 3 and 6 to 12 years, respectively. ${ }^{10}$ Another study conducted by Ohara et al. reported chest wall deformity in $63.6 \%$ of patients who underwent cartilage harvest before 10 years of age and in $20 \%$ of patients who were older than 10 years at the time of harvest. ${ }^{8}$ Harvesting, however, is ideally done prior to full skeletal maturity, ${ }^{7}$ and early enough to minimize the psychosocial consequences of childhood ostracization. ${ }^{4}$ Most surgeons recommend microtia reconstruction with autogenous cartilage between the ages of 6 and 10 years.

Limited research has investigated the utility of computed tomography (CT) imaging to help guide the timing of surgery.,11-15 Although these studies concluded that CT imaging may be useful in planning the timing of microtia reconstruction, the expense and exposure to ionizing radiation limit the widespread use of this imaging modality. Unlike CT imaging, ultrasonography is relatively inexpensive and does not expose patients to radiation. A few studies have reported using ultrasound as an effective diagnostic tool for evaluating costochondral cartilage deformities and traumatic rib fractures, as well as for assessing calcification patterns in costal cartilage prior to rib graft harvesting for revision rhinoplasty. ${ }^{16-18}$ However, to our knowledge, there have no reports in the literature that describe the use of ultrasound for determining the optimal timing of microtia reconstruction. In this report, we describe a novel application of ultrasonography to evaluate costochondral cartilage for use in microtia repair.

\section{METHODS}

A 9-year-old male with right-sided microtia was identified prior to stage I of microtia reconstruction. Preoperative ultrasonography of the chest wall was performed using an Acuson S3000 ultrasound system and a 9L4 high-resolution linear transducer (Siemens Medical Solutions USA, Malvern, PA). Transverse and longitudinal static and dynamic images of the sixth through ninth distal rib cartilages were obtained. Maximum transverse and longitudinal lengths of each individual cartilage were measured. When the length of the 


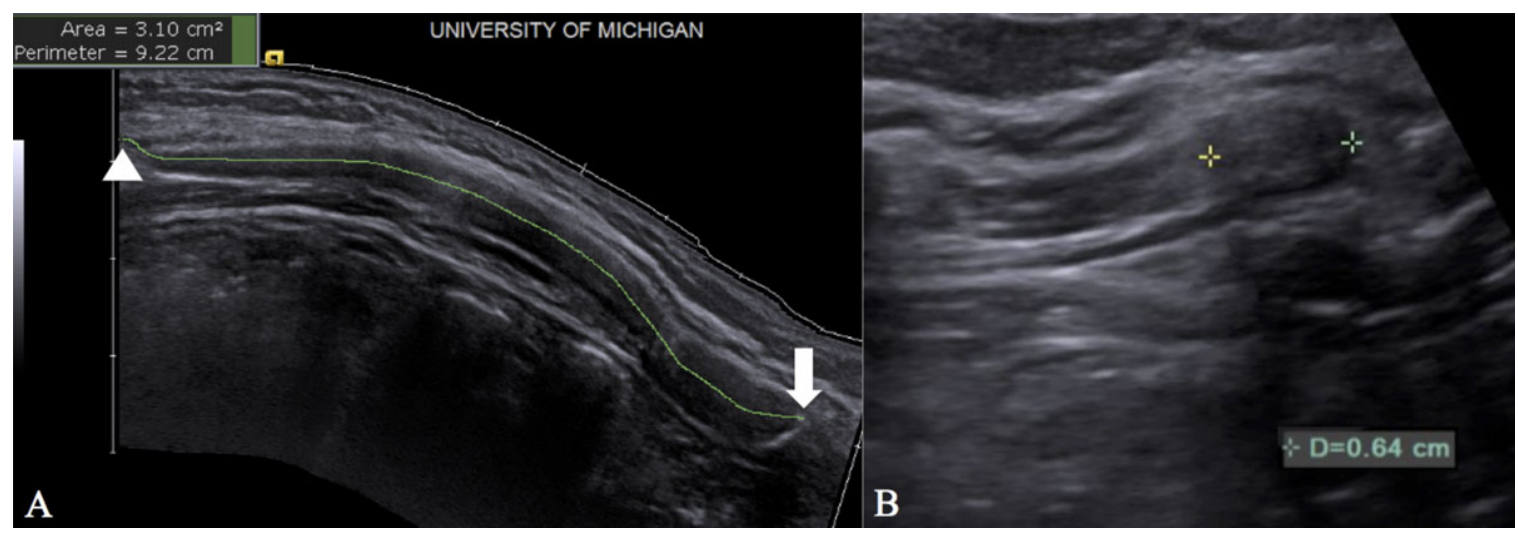

Fig. 1. Longitudinal panoramic (A) and standard transverse (B) sonographic images of the eighth rib distal cartilage. The costochondral junction (arrow) is identified as the interface between hyperechoic distal ossified rib and hypoechoic nonossified cartilage (arrow). The distal tip of the nonossified cartilage (arrowhead) is also identified for longitudinal cartilage measurement (green line on image A). The maximum cartilage transverse diameter is measured on a transverse view ( + and + on image $B$ ).

cartilage exceeded the length of the surface of the transducer, SieScape (Siemens Medical Solutions USA) panoramic real time images were acquired to improve anatomical information and to allow accurate measurements (Fig. 1). Intraoperatively, pictures were taken of the cartilaginous portion of the sixth through ninth ribs after harvest and prior to reconstruction, and rib dimensions were measured (Fig. 2). The rib dimensions obtained from the preoperative ultrasound were then compared with intraoperative rib measurements.

\section{RESULTS}

At the time of surgery, the patient was 9 years old and had a chest circumference that measured $69.5 \mathrm{~cm}$. The costochondral cartilage was more hypoechoic than the bony rib, which allowed for visualization of the costochondral junction. The transverse and longitudinal costochondral cartilage lengths were clearly visualized and measured on ultrasonography, and these measurements were compared to the actual rib measurements (Table I). All of the ultrasound measurements were within $0.4 \mathrm{~cm}$ of the harvested specimen measurements.

\section{DISCUSSION}

In determining the optimal timing for microtia reconstruction, surgeons must find a balance between operating too early, thus risking insufficient costochondral cartilage available for harvest, and delaying the surgery too long, potentially resulting in adverse psychosocial consequences. ${ }^{4,8-10}$ Currently, most surgeons who perform autogenous rib cartilage ear reconstruction begin this procedure between the ages of 6 and 10 years of age, depending on which technique is employed. ${ }^{19}$ Brent reconstructions are performed in four stages, with the first stage typically starting when a child is 6 years old. ${ }^{20}$ Nagata reconstructions require only two stages and begin at approximately 10 years of age, when the ear is approximately adult-sized and the chest wall circumference at the xiphoid is at least $60 \mathrm{~cm} .{ }^{19}$ Nagata reconstructions are performed later because they require significantly more cartilage for the antihelix, antitragal, and tragal complex, as well as an elevation piece for the second stage of the procedure. ${ }^{12}$ Although chest wall circumference can be a useful metric in some patients to determine the amount of costochondral cartilage available for harvest, its correlation to useable cartilage volume is not always reliable due to anatomical variability, childhood obesity, prior chest wall injury, and differences in overall nutritional status.

When performing a microtia reconstruction, it is not only the quantity of cartilage available for harvest that is important, but also the thickness of the rib cartilage used for the auricle framework. For the patient included in this study, measurements of cartilage thickness were not obtained intraoperatively, and thus we were unable to assess the accuracy of ultrasonography in measuring this dimension. However, because the longitudinal and transverse ultrasonographic measurements were similar to the actual measurements (all within $0.4 \mathrm{~cm}$ ), we anticipate that the ultrasonographic measurements of cartilage thickness will be of similar accuracy.

We investigated the utility of ultrasonography in determining the optimal timing for microtia reconstruction

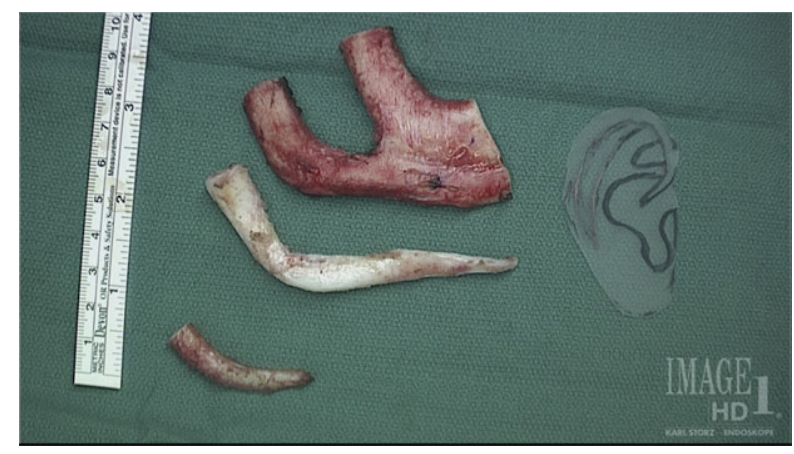

Fig. 2. Harvested costochondral cartilage from the sixth, seventh, eighth, and ninth ribs (superior to inferior). 
TABLE I.

Measurements of Rib Cartilage From Ultrasonography and Harvested Specimens

\begin{tabular}{lccc}
\hline Rib No. & $\begin{array}{c}\text { Ultrasonographic } \\
\text { Longitudinal Length }(\mathrm{cm})\end{array}$ & $\begin{array}{c}\text { Harvested Specimen } \\
\text { Longitudinal Length }(\mathrm{cm})\end{array}$ & $\begin{array}{c}\text { Ultrasonographic Transverse } \\
\text { Length (cm) }\end{array}$ \\
\hline 6 & 4.6 & 5.0 & $\begin{array}{c}\text { Harvested Specimen } \\
\text { Transverse Length }(\mathrm{cm})\end{array}$ \\
7 & 8.4 & 8.2 & 1.1 \\
8 & 9.2 & 9.5 & 1.0 \\
9 & 4.6 & 4.2 & 0.9 \\
\hline \hline
\end{tabular}

and demonstrated that it can accurately determine the dimensions of costochondral cartilage. In comparison to preoperative CT imaging, ultrasound is very inexpensive and does not expose patients to radiation. It is also relatively quick and does not require sedation. At our institution, we have developed a protocol allowing ultrasound to be obtained the same day of the child's preoperative appointment, providing significant convenience for families. At this preoperative appointment, we have started routinely collecting longitudinal, transverse, and thickness measurements of the costochondral cartilage.

For these reasons, high-resolution ultrasonography is a promising, novel approach to assess costochondral cartilage dimensions preoperatively. This noninvasive, low-risk modality may allow for a more accurate assessment of donor rib cartilage volume. Our group is currently assessing if this approach may translate to the ability to offer ear reconstruction earlier with greater assuredness that adequate donor cartilage is present in certain instances and avoid rare situations where there is an unexpected paucity of donor cartilage.

We are currently accruing a larger number of patients with ultrasonographic data at the time of the initial reconstructive surgery to determine optimal rib dimensions. Future research could investigate the interoperator variability with ultrasound assessment of costochondral cartilage.

\section{CONCLUSION}

Preoperative methods to accurately assess the amount of costochondral cartilage available for harvest in microtia reconstruction are lacking. We propose a lowcost, virtually risk-free preoperative technique for providing detailed rib dimensions. High-resolution ultrasonography has the potential to allow surgeons to evaluate rib dimensions prior to microtia reconstruction with greater accuracy, permitting safe and effective microtia repair earlier in a child's development.

\section{BIBLIOGRAPHY}

1. Luquetti DV, Heike CL, Hing AV, Cunningham ML, Cox TC. Microtia: epidemiology and genetics. Am J Med Genet A 2012;158A:124-139.

2. Brent B. Microtia repair with rib cartilage grafts: a review of personal experience with 1000 cases. Clin Plast Surg 2002;29:257-271, vii.

3. Horlock N, Vogelin E, Bradbury ET, Grobbelaar AO, Gault DT. Psychosocial outcome of patients after ear reconstruction: a retrospective study of 62 patients. Ann Plast Surg 2005;54:517-524.

4. Steffen A, Wollenberg B, Konig IR, Frenzel H. A prospective evaluation of psychosocial outcomes following ear reconstruction with rib cartilage in microtia. J Plast Reconstr Aesthet Surg 2010;63:1466-1473.

5. Jiamei D, Jiake C, Hongxing Z, Wanhou G, Yan W, Gaifen L. An investigation of psychological profiles and risk factors in congenital microtia patients. J Plast Reconstr Aesthet Surg 2008;61(suppl 1):S37-S43.

6. Tanzer RC. Total reconstruction of the external auricle. Arch Otolaryngol 1961;73:64-68.

7. Patel SA, Bhrany AD, Murakami CS, Sie KC. Autologous costochondral microtia reconstruction. Facial Plast Surg 2016;32:188-198.

8. Ohara K, Nakamura K, Ohta E. Chest wall deformities and thoracic scoliosis after costal cartilage graft harvesting. Plast Reconstr Surg 1997;99:1030-1036.

9. Sun Z, Yu X, Chen W, et al. Costal cartilage assessment in surgical timing of microtia reconstruction. J Craniofac Surg 2017;28:1521-1525.

10. Thomson HG, Kim TY, Ein SH. Residual problems in chest donor sites after microtia reconstruction: a long-term study. Plast Reconstr Surg 1995;95: 961-968.

11. Andreoli SM, Mills JC, Kilpatrick LA, White DR, Patel KG. CT measured normative cartilage growth in children requiring costochondral grafting. Otolaryngol Head Neck Surg 2013;149:924-930.

12. Moon IY, Oh KS, Lim SY, Pyon JK, Mun GH, Bang SI. Estimation of eighth costal cartilage in surgical timing of microtia reconstruction. $J$ Craniofac Surg 2015;26:48-51.

13. Kim H, Hwang JH, Lim SY, et al. Preoperative rib cartilage imaging in 3-dimensional chest computed tomography for auricular reconstruction for microtia. Ann Plast Surg 2014;72:428-434.

14. Wang Y, He L, Liu L, Chen W, Jiang H, Shang Q. Evaluation of multi-slice spiral ct scan and image reconstruction technology in estimating costal cartilage volume [in Chinese]. Zhongguo Xiu Fu Chong Jian Wai Ke Za Zhi 2014;28:1266-1269.

15. Kang SS, Guo Y, Zhang DY, Jiang DY. Rib cartilage assessment relative to the healthy ear in young children with microtia guiding operative timing. Chin Med J (Engl) 2015;128:2208-2214.

16. Supakul N, Karmazyn B. Ultrasound evaluation of costochondral abnormalities in children presenting with anterior chest wall mass. AJR Am J Roentgenol 2013;201:W336-W341.

17. Turk F, Kurt AB, Saglam S. Evaluation by ultrasound of traumatic rib fractures missed by radiography. Emerg Radiol 2010;17:473-477.

18. Bozzato A, Bumm K, Hertel V, Wurm J. Ultrasonographic evaluation of calcification patterns in costal cartilage: implications for rib graft harvesting. JAMA Facial Plast Surg 2013;15:457-460.

19. Yamada A. Autologous rib microtia construction: Nagata technique. Facial Plast Surg Clin North Am 2018;26:41-55.

20 . Brent B. The correction of microtia with autogenous cartilage grafts: I. the classic deformity. Plast Reconstr Surg 1980;66:1-12. 